\title{
In systemic sclerosis TAPSE/sPAP ratio is correlated with ventilatory efficiency and exercise capacity assessed by CPET
}

\author{
Amalia Colalillo $^{1} \cdot$ Chiara Pellicano $^{1} \cdot$ Antonella Romaniello $^{2} \cdot$ Edoardo Rosato $^{1}$ (i)
}

Received: 21 December 2021 / Accepted: 3 February 2022

(c) The Author(s) 2022

\begin{abstract}
Objectives The aim of the study was to evaluate the correlation between cardiopulmonary exercise testing (CPET) parameters and right ventricular echocardiographic parameters for pulmonary arterial hypertension screening in a cohort of systemic sclerosis (SSc) patients. Methods forty SSc patients were examined using CPET and resting transthoracic echocardiography. CPET parameters analyzed were minute ventilation/carbon dioxide production $\left(\mathrm{VE} / \mathrm{VCO}_{2}\right)$ slope and maximum oxygen uptake $\left(\mathrm{VO}_{2} \mathrm{max}\right)$; echocardiographic parameters were systolic pulmonary artery pressure (sPAP), tricuspid annular plane systolic excursion (TAPSE), and TAPSE/sPAP ratio. Results a positive correlation was observed between $\mathrm{VE} / \mathrm{VCO}_{2}$ slope and age $(r=0.415, p<0.01)$ and $\operatorname{sPAP}(r=0.461, p<0.01)$, conversely, a negative correlation was found between $\mathrm{VE} / \mathrm{VCO}_{2}$ slope and TASPE/sPAP ratio $(r=-0.521, p=0.001) . \mathrm{VO}_{2}$ max showed an inverse correlation with age $(r=-0.367, p<0.05)$ and SPAP $(r=-0.387, p<0.05)$ and a positive correlation with TAPSE/sPAP ratio $(r=0.521, p<0.01)$. On stepwise linear regression analysis, $\mathrm{VE} / \mathrm{VCO}_{2}$ slope was significantly correlated with TAPSE/sPAP ratio $(\beta$ coefficient $=-0.570 ; p<0.0001)$, as well as $\mathrm{VO}_{2}$ max was significantly correlated with TAPSE/sPAP ratio $(\beta$ coefficient $=0.518 ; p=0.001)$. Conclusion in SSc patients, TAPSE/sPAP ratio is the echocardiographic parameter of RV function which showed the best correlation with ventilatory efficiency and exercise capacity.
\end{abstract}

Keywords Systemic sclerosis $\cdot$ Echocardiography $\cdot$ TAPSE/sPAP ratio $\cdot$ Cardiopulmonary exercise testing $\cdot$ Pulmonary hypertension

\section{Introduction}

Pulmonary arterial hypertension (PAH) is characterized by progressive pulmonary vascular remodeling resulting in increased pulmonary vascular resistance and pulmonary arterial pressure, eventually leading to right ventricular (RV) failure [1]. PAH is a leading cause of morbidity and mortality in systemic sclerosis (SSc) patients [2]. Early diagnosis and treatment have been associated with improved outcomes $[3,4]$. Annual screening with echocardiography, diffusing capacity of the lung for carbon monoxide $\left(\mathrm{DL}_{\mathrm{CO}}\right)$ and natriuretic peptides is recommended by current guidelines. Right heart catheterization (RHC) is required to confirm

Edoardo Rosato

edoardo.rosato@uniroma1.it

1 Department of Translational and Precision Medicine, Sapienza University of Rome, Viale dell'Università 37, 00185 Rome, Italy

2 Division of Cardiology, Sant'Andrea Hospital, Rome, Italy
PAH diagnosis and to assess the severity of haemodynamic impairment [5]. Cardiopulmonary exercise testing (CPET) has been included in PAH diagnostic algorithm in order to reduce unnecessary invasive procedures $[6,7]$.

CPET is a safe non-invasive, dynamic technique providing an integrative assessment of cardiovascular, respiratory, metabolic, and muscular response to physical effort. Among CPET parameters, maximum oxygen uptake $\left(\mathrm{VO}_{2} \max \right)$ and minute ventilation/carbon dioxide production $\left(\mathrm{VE} / \mathrm{VCO}_{2}\right)$ slope have been the most widely studied in cardiopulmonary diseases and have proved to be useful not only for PAH detection but also as prognostic markers [8, 9]. A low $\mathrm{VO}_{2}$ max is an index of reduced exercise capacity. A high VE/ $\mathrm{VCO}_{2}$ slope reflects reduced ventilatory efficiency and is a hallmark of pulmonary vascular diseases [10].

Pressure overload is the main cause of RV dysfunction and failure in PAH. The RV-pulmonary arterial (PA) coupling describes the continuum of RV adaptation to pulmonary arterial load. The tricuspid annular plane systolic excursion/systolic pulmonary artery pressure (TAPSE/sPAP) 
ratio is the echocardiographic estimate of RV-PA coupling measured invasively by RHC [11]. A reduced TAPSE/sPAP ratio has been associated with poor outcomes in patients with PAH [12]. TAPSE/sPAP ratio is a powerful predictor of all-cause mortality in patients with moderate or severe tricuspid regurgitation [13]. Moreover, it has recently been demonstrated the potential role of TAPSE/sPAP ratio in SScPAH diagnosis [14].

An association between RV function parameters and VE/ $\mathrm{VCO}_{2}$ slope and $\mathrm{VO}_{2}$ max has already been reported in heart failure and PAH $[15,16]$. No study to date has specifically investigated the relationship between CPET measures and TAPSE/sPAP ratio.

The aim of the study was to evaluate the correlation between CPET parameters and RV echocardiographic parameters for PAH screening in a cohort of SSc patients.

\section{Methods}

\section{Subjects}

Forty patients meeting the 2013 American College of Rheumatology/European League against Rheumatism classification criteria for SSc [17] were enrolled in the study. Diffuse cutaneous SSc (dcSSc) and limited cutaneous SSc (lcSSc) were classified according to Le Roy et al. [18].

Patients with unstable angina, heart failure, arrhythmias, neurological disorders, valvular heart disease, uncontrolled arterial hypertension, hypertrophic cardiomyopathy, compromised exercise performance, mental or cognitive impairment, peripheral vascular diseases, anaemia, pregnant or breastfeeding women and subjects unable to give written informed consent were excluded. Calcium channel blockers, endotelin-1 receptor antagonist, and phosphodiesterase type 5 inhibitors were interrupted $72 \mathrm{~h}$ before CPET examination. CPET was performed $24 \mathrm{~h}$ prior to the next infusion of Iloprost.

The subjects' written consent was obtained according to the declaration of Helsinki and the study was approved by the ethics committee of Sapienza University.

\section{Echocardiography}

Resting transthoracic echocardiography was performed in all SSc patients by the same experienced operator with the General Electric Vivid S5 apparatus (GE Medical Systems, Israel Ltd.). Left ventricle (LV) diameter, wall thickness, LV ejection fraction (EF), RV diameter, TAPSE, left and right atrium area were assessed by standard methods [19]. sPAP was calculated from peak tricuspidal jet velocity using the simplified Bernoulli equation and combining this value with an estimate of the right atrium (RA) pressure: $\mathrm{sPAP}=4(V)^{2}+\mathrm{RA}$ pressure, where $V$ is the peak velocity (in meters per second) of the tricuspid valve regurgitant jet, and RA pressure is estimated from inferior vena cava (IVC) diameter and respiratory changes [20].

\section{CPET}

An incremental symptom-limited CPET was performed on an electronically braked cycloergometer (Ergoline-800, Mortara, Bologna, Italy), according to our previous study [21]. The subject was connected to the breath-by-breath lung gas exchange system by the use of a mask and breathing through a bidirectional turbine mass flow sensor (Quark PFT, Cosmed, Rome, Italy). The exercise protocol consisted of $3 \mathrm{~min}$ of rest and $3 \mathrm{~min}$ of unloaded cycling, followed by an incremental work rate to induce voluntary exhaustion in about $10 \mathrm{~min}$, followed by $3 \mathrm{~min}$ of recovery. ECG and pulse oximetry were continuously monitored, and blood pressure was measured every two minutes. $\mathrm{VO}_{2}, \mathrm{VCO}_{2}$ and the respiratory exchange ratio $\left(\mathrm{VCO}_{2} / \mathrm{VO}_{2}, \mathrm{RER}\right)$ were computed and averaged every $10 \mathrm{~s}$. The anaerobic threshold (AT) was determined by the $V$-slope method. The relation between VE and $\mathrm{VCO}_{2}\left(\mathrm{VE} / \mathrm{VCO}_{2}\right.$ slope $)$ was calculated as the slope of the linear relationship between $\mathrm{VE}$ and $\mathrm{VCO}_{2}$ from one minute after the beginning of the loaded exercise to the end of the isocapnic buffering period. A submaximal test is defined by RER $\leq 1.05$.

\section{Statistical analysis}

All results are expressed as the median and interquartile range (IQR), SPSS version 26.0 software was used for statistical analysis. The Shapiro-Wilk test was used to evaluate the normal distribution of data. Group of comparisons were made by Mann-Whitney test. The Fisher's exact test was used to compare categorical variables. Spearman coefficient was used to evaluate the linear correlation. Stepwise regression analysis with significant variables in linear correlation was used to evaluate the correlation between the dependent variable (VE/VCO2 slope and $\mathrm{VO} 2 \mathrm{max}$ ) and independent variables. $P$-values $<0.05$ were considered significant.

\section{Results}

Forty SSc patients [35 females (87.5\%); median age 53 (IQR 46-59) years] were enrolled in the study. Twenty-three patients (57.5\%) had lcSSc. Clinical, echocardiographic and CPET parameters of SSc patients are reported in Table 1.

$\mathrm{VE} / \mathrm{VCO}_{2}$ slope and $\mathrm{VO}_{2}$ max median values were 29.2 (IQR 25.3-32.7) and 19.7 (IQR 17.85-24.21) mL/min/kg, 
Table 1 Clinical, echocardiographic and cardiopulmonary exercise testing (CPET) parameters of systemic sclerosis (SSc) patients

\begin{tabular}{|c|c|}
\hline \multicolumn{2}{|l|}{ Demographic and clinical features } \\
\hline Female, $n(\%)$ & $35(87.5)$ \\
\hline Age, years & $53(46-59)$ \\
\hline BMI, kg/m² & $22.1(20.4-24.5)$ \\
\hline lcSSc, $n(\%)$ & $23(57.5)$ \\
\hline Disease duration, years & $11(6-18)$ \\
\hline \multicolumn{2}{|l|}{ SSc-specific autoantibodies, $n(\%)$} \\
\hline Scl70 & $17(42.5)$ \\
\hline Anti-centromere & $19(47.5)$ \\
\hline None & $4(10)$ \\
\hline \multicolumn{2}{|l|}{ Capillaroscopic pattern, $n(\%)$} \\
\hline Early & $6(15)$ \\
\hline Active & $13(32.5)$ \\
\hline Late & $21(52.5)$ \\
\hline mRSS & $11(7-15)$ \\
\hline DAI & $1.5(0.8-2.3)$ \\
\hline DSS & $4(3-6)$ \\
\hline FVC, $\%$ of predicted value & $102(92-108)$ \\
\hline DLco, $\%$ of predicted value & $73(68-83)$ \\
\hline \multicolumn{2}{|l|}{ Echocardiographic parameters } \\
\hline Ejection fraction, $\%$ & $60(60-63)$ \\
\hline Right atrium area, $\mathrm{cm}^{2}$ & $13.5(9-19)$ \\
\hline $\mathrm{TRV}, \mathrm{m} / \mathrm{s}$ & $2.21(1.53-3.68)$ \\
\hline TAPSE, mm & $24(22-27)$ \\
\hline sPAP, mmHg & $28(25-32)$ \\
\hline TAPSE/sPAP mm/mmHg & $0.87(0.75-1.05)$ \\
\hline \multicolumn{2}{|l|}{ CPET parameters } \\
\hline RER & $1.22(1.14-1.31)$ \\
\hline Maximum workload, W & $80(60-100)$ \\
\hline $\mathrm{VO}_{2} \max , \mathrm{mL} / \mathrm{kg} / \mathrm{min}$ & $19.7(17.85-24.21)$ \\
\hline $\mathrm{VO}_{2} \max , \%$ of predicted value & $81(73-87)$ \\
\hline $\mathrm{VO}_{2} @ \mathrm{AT}, \mathrm{mL} / \mathrm{min}$ & $739(651-887)$ \\
\hline $\mathrm{VE} / \mathrm{VCO}_{2}$ slope & $29.2(25.3-32.7)$ \\
\hline $\mathrm{PetCO}_{2}$ max, mmHg & $35(33-38)$ \\
\hline HR rest, bpm & $79(72-84)$ \\
\hline HR max, bpm & $155(138-167)$ \\
\hline $\mathrm{SpO} 2 \max , \%$ & 98 (96-99) \\
\hline
\end{tabular}

Values are reported as median (interquartile range). BMI Body mass index; $l c S S c$ Limited cutaneous systemic sclerosis; Scl70 Antitopoisomerase I antibodies; $m R S S$ Modified Rodnan skin score; DAI Disease activity index; DSS Disease severity score. FVC Forced vital capacity; DLco Diffusing capacity of the lungs for carbon monoxide; TRV Tricuspid regurgitation velocity; TAPSE Tricuspid annular plane systolic excursion; $S P A P$ Systolic pulmonary arterial pressure; $R E R$ Respiratory exchange ratio; $\mathrm{VO}_{2}$ max Maximum oxygen uptake; $V O_{2} @ A T \mathrm{VO}_{2}$ at anaerobic threshold; VE/VCO 2 Minute ventilation/ carbon dioxide production; $\mathrm{PetCO}_{2}$ End-tidal carbon dioxide; $\mathrm{HR}$ Heart rate; $\mathrm{SpO}_{2}$ Arterial oxygen saturation

respectively. sPAP and TAPSE/sPAP ratio median value were 28 (25-32) $\mathrm{mmHg}$ and 0.87 (IQR $0.75-1.05) \mathrm{mm} /$ $\mathrm{mmHg}$, respectively.
A significant positive correlation was found between $\mathrm{VE} / \mathrm{VCO}_{2}$ slope and age $(r=0.415, p<0.01)$ and sPAP $(r=0.461, p<0.01)$, conversely, a significant negative correlation was observed between VE/ $\mathrm{VCO}_{2}$ slope and TAPSE/ sPAP ratio $(r=-0.521, p=0.001)$ (Fig. 1). No correlation was found between $\mathrm{VE} / \mathrm{VCO}_{2}$ slope and TAPSE. By stepwise linear regression analysis, $\mathrm{VE} / \mathrm{VCO}_{2}$ slope was significantly correlated with TAPSE/sPAP ratio ( $\beta$ coefficient $=-0.570 ; p<0.0001$ ).

$\mathrm{VO}_{2}$ max showed a significant negative correlation with age $(r=-0.367, p<0.05)$ and $\operatorname{sPAP}(r=-0.387, p<0.05)$ and a significant positive correlation with TAPSE/sPAP ratio $(r=0.521, p<0.01)$ (Fig. 1$)$. No correlation was found between $\mathrm{VO}_{2}$ max and TAPSE. On stepwise regression analysis $\mathrm{VO}_{2}$ max was significantly correlated with TAPSE/ sPAP ratio $(\beta$ coefficient $=0.518 ; p=0.001)$.

\section{Discussion}

In this study, $\mathrm{VE} / \mathrm{VCO}_{2}$ slope and $\mathrm{VO}_{2}$ max showed a correlation with age, sPAP and TAPSE/sPAP ratio. Therefore, in multiple regression analysis TAPSE/sPAP ratio is the only variable, which shows a significant correlation with exercise capacity $\left(\mathrm{VO}_{2} \max \right)$ and ventilatory efficiency $\left(\mathrm{VE} / \mathrm{VCO}_{2}\right.$ slope).

Reducing the time to PAH diagnosis can significantly improve patients' survival. Current guidelines recommend resting echocardiography as a screening test in SSc patients. Although elevated sPAP values are associated with an increased probability of PAH, sPAP estimation may be inaccurate in the individual patient. In the ESC/ERS guidelines, CPET was performed to reduce unnecessary invasive procedures and in PAH risk assessment [5].

In our study, we demonstrated that TAPSE/sPAP ratio is the best echocardiographic parameter of RV function which correlates with $\mathrm{VE} / \mathrm{VCO}_{2}$ slope and $\mathrm{VO}_{2}$ max. In clinical practice, sPAP is the most used echocardiograpic parameter to evaluate PAH. In our previous study, we demonstrated that in SSc patients with a DETECT algorithm step 2 total score $>35$ TAPSE/sPAP ratio can be used to further select patients requiring RHC to confirm PAH diagnosis [14]. Dumitrescu et al. investigated the role of CPET in early PAH detection in a prospective study of $173 \mathrm{SSc}$ patients, without known PAH, who also underwent RHC. PeakVO 2 and VE/ $\mathrm{VCO}_{2}$ were the CPET parameters, which showed the highest correlations with pulmonary haemodynamics. PeakVO $\mathrm{V}_{2}$ was the most accurate parameter for diagnosis (sensitivity $87.5 \%$, specificity $74.8 \%$ at a threshold level of $13.8 \mathrm{~mL} / \mathrm{min} / \mathrm{kg}$ ) and a peakVO $\mathrm{V}_{2}$ of $>18.7 \mathrm{~mL} / \mathrm{kg} / \mathrm{min}$ excluded PAH (negative predictive value of 1.0). In addition, a nadir $\mathrm{VE} / \mathrm{VCO}_{2}$ ratio of $>45.5$ showed a positive predictive value of 1.0 [6]. Santaniello et al. demonstrated that CPET is useful, in addition to the DETECT 

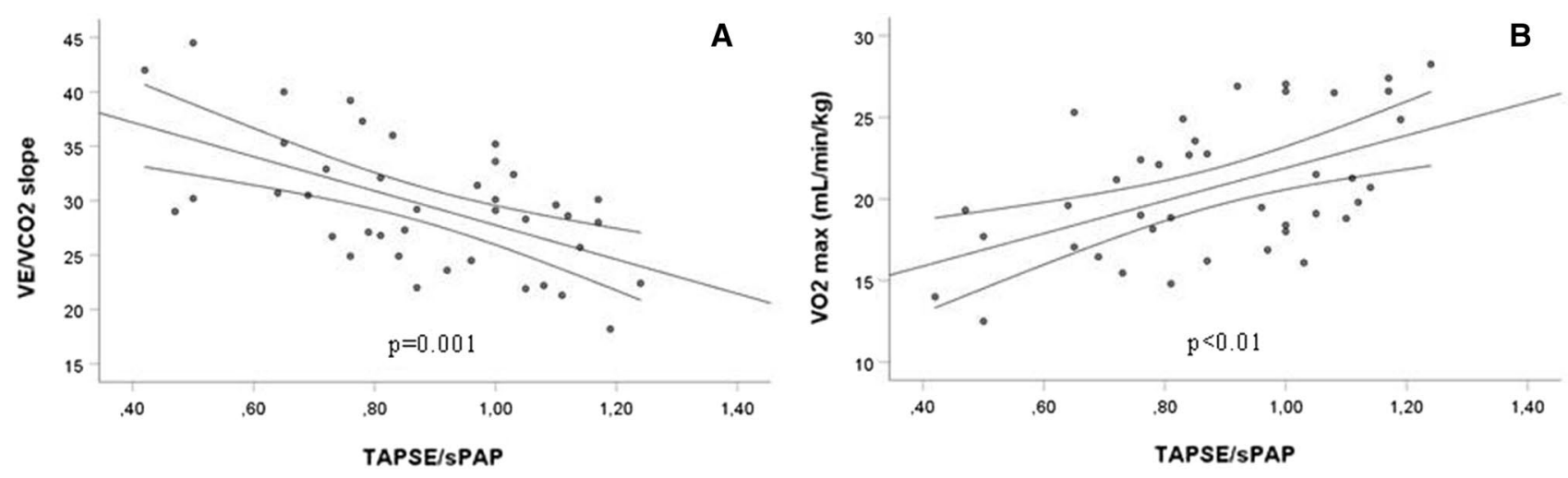

Figure 1 A Correlation between minute ventilation/carbon dioxide production (VE/ $\left.\mathrm{VCO}_{2}\right)$ slope and tricuspid annular plane systolic excursion/ systolic pulmonary artery pressure (TAPSE/sPAP) ratio; B Correlation between maximum oxygen uptake $\left(\mathrm{VO}_{2}\right.$ max) and TAPSE/sPAP ratio

algorithm, to select patients for RHC referral. Among CPET parameters $\mathrm{VE} / \mathrm{VCO}_{2}$ slope showed the best performance to predict PAH at RHC [7]. An association between RV function parameters and $\mathrm{VE} / \mathrm{VCO}_{2}$ slope and $\mathrm{VO}_{2}$ max has already been reported. In heart failure, higher SPAP and lower RV fractional area change (FAC) were correlated with worse percent predicted $\mathrm{VO}_{2}$ max and higher $\mathrm{VE} / \mathrm{VCO}_{2}$ slope [15]. In $\mathrm{PAH}$, resting RV function assessed by FAC or RV longitudinal strain was also associated with the CPET parameters of exercise capacity and ventilatory efficiency [16]. To date, no study has investigated the correlation between TAPSE/sPAP ratio and CPET parameters. We can assume that TAPSE/sPAP ratio could be a useful echocardiographic parameter of RV function to select SSc patients requiring CPET. The main limitations of our study are the small sample size, the lack of a control group, the lack of RHC data and the prospective role of TAPSE/sPAP ratio during the follow-up of SSc patients.

In conclusion, in SSc patients TAPSE/sPAP ratio is the echocardiographic parameter of RV function which showed the best correlation with ventilatory efficiency and exercise capacity. Future larger studies are needed to confirm our preliminary data.

Funding The authors declare that no funds, grants, or other support were received during the preparation of this manuscript.

\section{Declarations}

Conflict of interest The authors have no relevant financial or non-financial interests to disclose.

Open Access This article is licensed under a Creative Commons Attribution 4.0 International License, which permits use, sharing, adaptation, distribution and reproduction in any medium or format, as long as you give appropriate credit to the original author(s) and the source, provide a link to the Creative Commons licence, and indicate if changes were made. The images or other third party material in this article are included in the article's Creative Commons licence, unless indicated otherwise in a credit line to the material. If material is not included in the article's Creative Commons licence and your intended use is not permitted by statutory regulation or exceeds the permitted use, you will need to obtain permission directly from the copyright holder. To view a copy of this licence, visit http://creativecommons.org/licenses/by/4.0/.

\section{References}

1. Humbert M, Guignabert C, Bonnet S, et al. Pathology and pathobiology of pulmonary hypertension: state of the art and research perspectives. Eur Respir J. 2019;53(1):1801887. https://doi.org/ 10.1183/13993003.01887-2018.

2. Elhai M, Meune C, Boubaya M, et al. Mapping and predicting mortality from systemic sclerosis. Ann Rheum Dis. 2017;76(11):1897-905. https://doi.org/10.1136/annrh eumdis-2017-211448.

3. Hughes M, Zanatta E, Sandler RD, Avouac J, Allanore Y. Improvement with time of vascular outcomes in systemic sclerosis: a systematic review and meta-analysis study. Rheumatology (Oxford). 2021. https://doi.org/10.1093/rheumatology/keab850.

4. Brown Z, Proudman S, Morrisroe K, Stevens W, Hansen D, Nikpour M. Screening for the early detection of pulmonary arterial hypertension in patients with systemic sclerosis: a systematic review and meta-analysis of long-term outcomes. Semin Arthritis Rheum. 2021;51(3):495-512. https://doi.org/10.1016/j.semarthrit. 2021.03.011.

5. Galiè N, Humbert M, Vachiery JL, et al. 2015 ESC/ERS Guidelines for the diagnosis and treatment of pulmonary hypertension: the joint task force for the diagnosis and treatment of pulmonary hypertension of the European society of cardiology (ESC) and the European respiratory society (ERS): endorsed by: association for European paediatric and congenital cardiology (AEPC), international society for heart and lung transplantation (ISHLT). Eur Heart J. 2016;37(1):67-119. https://doi.org/10.1093/eurheartj/ ehv317.

6. Dumitrescu D, Nagel C, Kovacs G, et al. Cardiopulmonary exercise testing for detecting pulmonary arterial hypertension in systemic sclerosis. Heart. 2017;103(10):774-82. https://doi.org/10. 1136/heartjnl-2016-309981. 
7. Santaniello A, Casella R, Vicenzi M, et al. Cardiopulmonary exercise testing in a combined screening approach to individuate pulmonary arterial hypertension in systemic sclerosis. Rheumatology (Oxford). 2020;59(7):1581-6. https://doi.org/10.1093/rheum atology/kez473.

8. Wensel R, Francis DP, Meyer FJ, et al. Incremental prognostic value of cardiopulmonary exercise testing and resting haemodynamics in pulmonary arterial hypertension. Int J Cardiol. 2013;167(4):1193-8. https://doi.org/10.1016/j.ijcard.2012.03.135.

9. Ewert R, Ittermann T, Habedank D, et al. Prognostic value of cardiopulmonary exercise testing in patients with systemic sclerosis. BMC Pulm Med. 2019;19(1):230. https://doi.org/10.1186/ s12890-019-1003-7.

10. Weatherald J, Philipenko B, Montani D, Laveneziana P. Ventilatory efficiency in pulmonary vascular diseases. Eur Respir Rev. 2021;30(161): 200214. https://doi.org/10.1183/16000617. 0214-2020.

11. Tello K, Wan J, Dalmer A, et al. Validation of the tricuspid annular plane systolic excursion/systolic pulmonary artery pressure ratio for the assessment of right ventricular-arterial coupling in severe pulmonary hypertension. Circ Cardiovasc Imaging. 2019;12(9): e009047. https://doi.org/10.1161/CIRCIMAGING. 119.009047.

12. Tello K, Axmann J, Ghofrani HA, et al. Relevance of the TAPSE/ PASP ratio in pulmonary arterial hypertension. Int J Cardiol. 2018;266:229-35. https://doi.org/10.1016/j.ijcard.2018.01.053.

13. Saeed S, Smith J, Grigoryan K, et al. The tricuspid annular plane systolic excursion to systolic pulmonary artery pressure index: association with all-cause mortality in patients with moderate or severe tricuspid regurgitation. Int J Cardiol. 2020;317:176-80. https://doi.org/10.1016/j.ijcard.2020.05.093.

14. Colalillo A, Grimaldi MC, Vaiarello V, et al. In systemic sclerosis TAPSE/sPAP ratio can be used in addition to the DETECT algorithm for pulmonary arterial hypertension diagnosis. Rheumatology (Oxford). 2021. https://doi.org/10.1093/rheumatology/keab7 48.

15. Teramoto K, Sengelov M, West E, et al. Association of pulmonary hypertension and right ventricular function with exercise capacity in heart failure. ESC Heart Fail. 2020;7(4):1635-44. https://doi. org/10.1002/ehf2.12717.
16. Rehman MB, Howard LS, Christiaens LP, Gill D, Gibbs JSR, Nihoyannopoulos P. Resting right ventricular function is associated with exercise performance in PAH, but not in CTEPH. Eur Heart J Cardiovasc Imaging. 2018;19(2):185-92. https://doi.org/ 10.1093/ehjci/jex002.

17. van den Hoogen F, Khanna D, Fransen J, et al. 2013 classification criteria for systemic sclerosis: an American college of rheumatology/European league against rheumatism collaborative initiative. Ann Rheum Dis. 2013;72(11):1747-55. https://doi.org/10.1136/ annrheumdis-2013-204424.

18. Lang RM, Bierig M, Devereux RB, et al. Recommendations for chamber quantification: a report from the American society of echocardiography's guidelines and standards committee and the chamber quantification writing group, developed in conjunction with the European association of echocardiography, a branch of the European society of cardiology. J Am Soc Echocardiogr. 2005;18(12):1440-63. https://doi.org/10.1016/j.echo.2005.10. 005.

19. LeRoy EC, Black C, Fleischmajer R, et al. Scleroderma (systemic sclerosis): classification, subsets and pathogenesis. J Rheumatol. 1988;15(2):202-5.

20. Rudski LG, Lai WW, Afilalo J, et al. Guidelines for the echocardiographic assessment of the right heart in adults: a report from the American society of echocardiography endorsed by the European association of echocardiography, a registered branch of the European society of cardiology, and the Canadian society of echocardiography. J Am Soc Echocardiogr. 2010;23(7):685-788. https://doi.org/10.1016/j.echo.2010.05.010.

21. Rosato E, Leodori G, Gigante A, Di Paolo M, Paone G, Palange $P$. Reduced ventilatory efficiency during exercise predicts major vascular complications and mortality for interstitial lung disease in systemic sclerosis. Clin Exp Rheumatol. 2020;38(Suppl 125):85-91.

Publisher's Note Springer Nature remains neutral with regard to jurisdictional claims in published maps and institutional affiliations. 